\title{
$2 \mathrm{Sr}_{5} \mathrm{Ga}_{8} \mathrm{O}_{3} \mathrm{~S}_{14}$ : A Nonlinear Optical Oxysulfide with 3 Melilite-Derived Structure and Wide Band Gap
}

4 Ruiqi Wang, ${ }^{\mathrm{a}}$ Yangwu Guo, ${ }^{\mathrm{b}}$ Xian Zhang, ${ }^{\mathrm{c}}$ Yi Xiao, ${ }^{\mathrm{a}}$ Jiyong Yao, ${ }^{*, \mathrm{~b}}$ Fuqiang Huang, ${ }^{*}, \mathrm{~d}, \mathrm{e}, \mathrm{e}$

$6{ }^{a}$ Beijing National Laboratory for Molecular Sciences and State Key Laboratory of Rare 7 Earth Materials Chemistry and Applications, College of Chemistry and Molecular $8 \quad$ Engineering, Peking University, Beijing 100871, P. R. China

$9{ }^{b}$ Beijing Center for Crystal Research and Development, Key Lab of Functional Crystals and 10 Laser Technology, Technical Institute of Physics and Chemistry, Chinese Academy of 11 Sciences Beijing, 100190 P. R. China

$12{ }^{c}$ Qian Xuesen Laboratory of Space Technology, China Academy of Space Technology, 13 Beijing, 100094 P. R. China

$14 d$ State Key Laboratory of High-Performance Ceramics and Superfine Microstructure, 15 Shanghai Institute of Ceramics, Chinese Academy of Sciences, Shanghai 200050, P. R. China

$16{ }^{e}$ CAS Center for Excellence in Superconducting Electronic (CENSE), Shanghai 200050, P. R. 17 China

18 *E-mail: jyao@mail.ipc.ac.cn

19 *E-mail: huangfq@pku.edu.cn 


\section{List of contents:}

2 1. Supplementary figures

3 2. Supplementary tables 4 


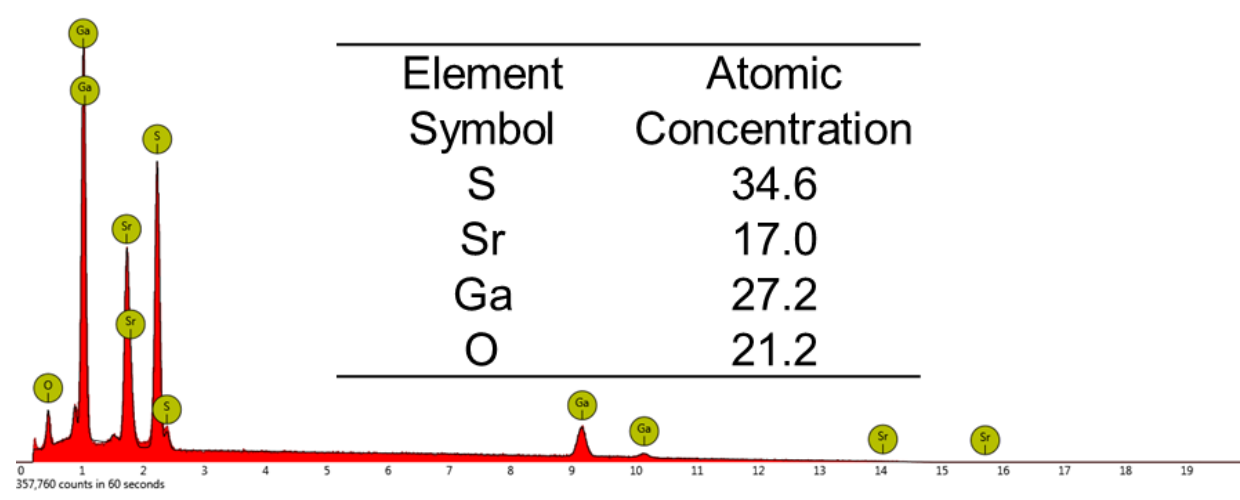

Figure S1 EDS spectrum and element contents of $\mathrm{Sr}_{5} \mathrm{Ga}_{8} \mathrm{O}_{3} \mathrm{~S}_{14}$. 


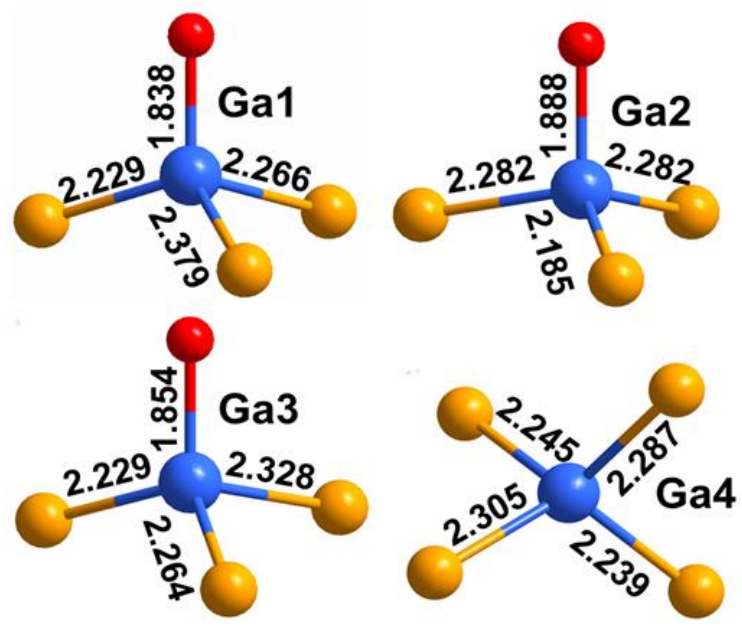

Figure S2. Coordination environments and bond lengths of Ga1, Ga2, Ga3 and Ga4. 


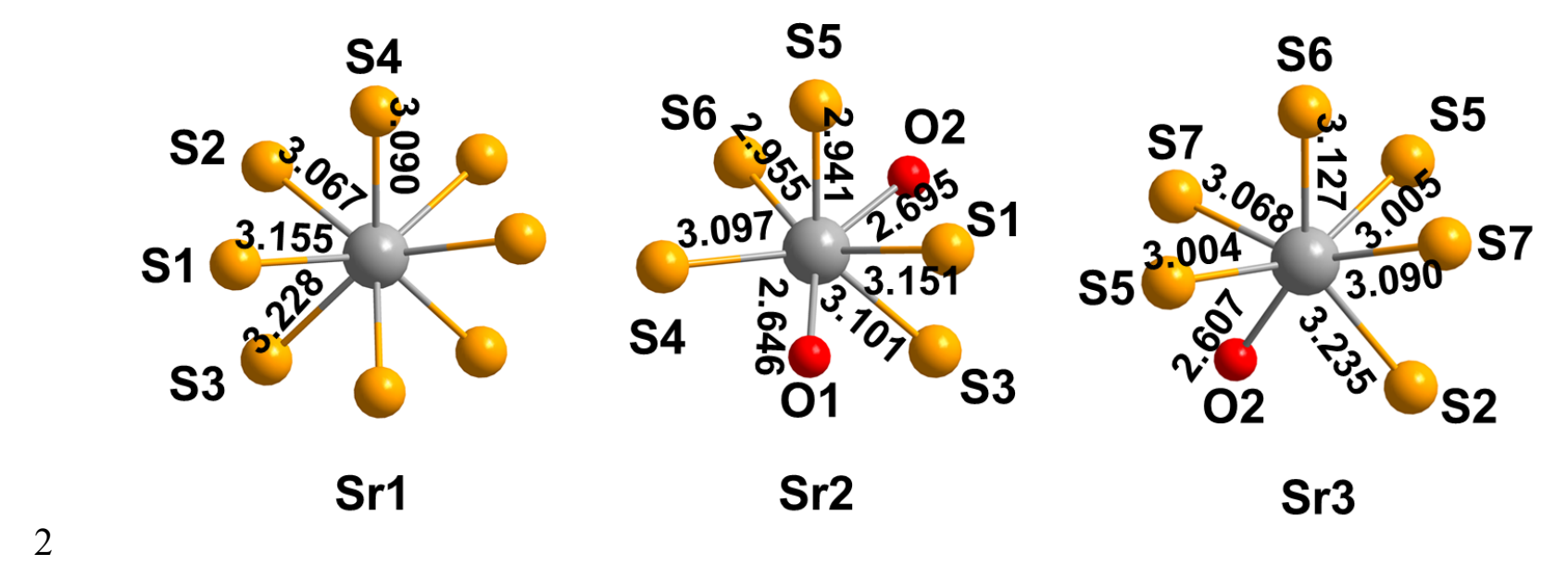

Figure S3. Coordination environments of $\mathrm{Sr} 1, \mathrm{Sr} 2$ and $\mathrm{Sr} 3$. 


\section{Supplementary tables.}

Table S1. Atomic coordinates and equivalent isotropic displacement parameters $\left(\AA^{2}\right)$ of $\mathrm{Sr}_{5} \mathrm{Ga}_{8} \mathrm{O}_{3} \mathrm{~S}_{14}$ at $180 \mathrm{~K}$

\begin{tabular}{|c|c|c|c|c|c|}
\hline Label & $\mathrm{x}$ & $\mathrm{y}$ & $\mathrm{z}$ & Occupancy & $\mathrm{U}_{\mathrm{eq}}{ }^{*}$ \\
\hline Sr1 & 1 & 0 & $0.48913(13)$ & 1 & $0.00594(18)$ \\
\hline $\mathrm{Sr} 2$ & $0.49609(7)$ & $0.09124(2)$ & $0.48840(9)$ & 1 & $0.00588(15)$ \\
\hline $\mathrm{Sr} 3$ & $0.80579(7)$ & $0.21853(2)$ & $0.47649(9)$ & 1 & $0.00612(15)$ \\
\hline Ga1 & $0.78551(8)$ & $0.10066(3)$ & $0.10117(11)$ & 1 & $0.00391(16)$ \\
\hline $\mathrm{Ga} 2$ & $0.52930(8)$ & $0.20088(3)$ & $0.06511(10)$ & 1 & $0.00390(17)$ \\
\hline $\mathrm{Ga} 3$ & $0.67858(7)$ & $-0.00272(3)$ & $0.87321(10)$ & 1 & $0.00371(16)$ \\
\hline $\mathrm{Ga} 4$ & $1.15161(7)$ & $0.14584(3)$ & $-0.00410(11)$ & 1 & $0.00489(17)$ \\
\hline S1 & $1.21408(18)$ & $-0.08065(6)$ & $0.7407(2)$ & 1 & $0.0046(3)$ \\
\hline S2 & $0.99465(19)$ & $0.11735(6)$ & $0.2583(2)$ & 1 & $0.0060(3)$ \\
\hline S3 & $0.70106(19)$ & $0.01077(6)$ & $0.2361(2)$ & 1 & $0.0048(3)$ \\
\hline S4 & $0.78953(18)$ & $-0.07911(6)$ & $0.7331(2)$ & 1 & $0.0051(3)$ \\
\hline S5 & $0.52851(18)$ & $0.20297(6)$ & $0.7101(2)$ & 1 & $0.0058(3)$ \\
\hline S6 & $0.32337(18)$ & $0.17201(7)$ & $0.2272(3)$ & 1 & $0.0075(3)$ \\
\hline S7 & $1.07176(18)$ & $0.21313(6)$ & $-0.2366(2)$ & 1 & $0.0065(3)$ \\
\hline $\mathrm{O} 1$ & 0.5 & 0 & $0.7471(9)$ & 1 & $0.0044(12)$ \\
\hline $\mathrm{O} 2$ & $0.6593(5)$ & $0.15316(16)$ & $0.2145(7)$ & 1 & $0.0053(9)$ \\
\hline
\end{tabular}

* $\mathrm{U}_{\text {eq }}$ is defined as one third of the trace of the orthogonalized $\mathrm{U}_{\mathrm{ij}}$ tensor. 
Table S2. Anisotropic displacement parameters $\left(\AA^{2}\right)$ of $\mathrm{Sr}_{5} \mathrm{Ga}_{8} \mathrm{O}_{3} \mathrm{~S}_{14}$ at $180 \mathrm{~K}$

\begin{tabular}{|c|c|c|c|c|c|c|}
\hline Label & $\mathrm{U}_{11}$ & $\mathrm{U}_{22}$ & $\mathrm{U}_{33}$ & $\mathrm{U}_{12}$ & $\mathrm{U}_{13}$ & $\mathrm{U}_{23}$ \\
\hline Sr1 & $0.0042(4)$ & $0.0055(4)$ & $0.0081(4)$ & 0 & 0 & $0.0005(3)$ \\
\hline $\mathrm{Sr} 2$ & $0.0059(3)$ & $0.0042(3)$ & $0.0075(3)$ & $0.0008(2)$ & $0.0021(2)$ & $-0.0004(2)$ \\
\hline $\mathrm{Sr} 3$ & $0.0048(3)$ & $0.0068(3)$ & $0.0068(3)$ & $-0.0015(2)$ & $-0.0001(2)$ & $-0.0017(2)$ \\
\hline Ga1 & $0.0041(4)$ & $0.0030(3)$ & $0.0047(3)$ & $-0.0009(3)$ & $-0.0001(3)$ & $0.0008(3)$ \\
\hline $\mathrm{Ga} 2$ & $0.0042(4)$ & $0.0035(3)$ & $0.0039(3)$ & $0.0006(2)$ & $0.0001(3)$ & $0.0013(3)$ \\
\hline Ga3 & $0.0031(4)$ & $0.0033(3)$ & $0.0047(3)$ & $-0.0009(3)$ & $-0.0008(3)$ & $0.0000(3)$ \\
\hline $\mathrm{Ga} 4$ & $0.0055(4)$ & $0.0039(3)$ & $0.0052(3)$ & $0.0003(3)$ & $0.0008(3)$ & $0.0005(3)$ \\
\hline S1 & $0.0052(8)$ & $0.0039(7)$ & $0.0047(7)$ & $0.0002(6)$ & $-0.0003(6)$ & $0.0000(6)$ \\
\hline S2 & $0.0046(8)$ & $0.0073(7)$ & $0.0063(7)$ & $0.0016(5)$ & $0.0007(7)$ & $-0.0008(7)$ \\
\hline S3 & $0.0054(8)$ & $0.0047(7)$ & $0.0044(7)$ & $-0.0002(6)$ & $0.0003(6)$ & $-0.0007(6)$ \\
\hline S4 & $0.0052(8)$ & $0.0039(7)$ & $0.0061(7)$ & $0.0009(6)$ & $0.0009(6)$ & $0.0024(6)$ \\
\hline S5 & $0.0065(9)$ & $0.0060(7)$ & $0.0050(6)$ & $0.0003(5)$ & $0.0001(6)$ & $0.0019(6)$ \\
\hline S6 & $0.0038(8)$ & $0.0100(8)$ & $0.0086(7)$ & $0.0006(6)$ & $-0.0014(6)$ & $-0.0014(6)$ \\
\hline S7 & $0.0078(8)$ & $0.0044(8)$ & $0.0072(7)$ & $0.0002(6)$ & $-0.0013(6)$ & $0.0004(6)$ \\
\hline $\mathrm{O} 1$ & $0.004(3)$ & $0.005(3)$ & $0.005(3)$ & 0 & 0 & $0.000(3)$ \\
\hline $\mathrm{O} 2$ & $0.004(3)$ & $0.004(2)$ & $0.009(2)$ & $-0.0009(16)$ & $-0.0003(17)$ & $0.0013(18)$ \\
\hline
\end{tabular}

